\title{
ExPLOSION IN A DIFFUSIVE STRIP DUE TO A CONCENTRATED NONLINEAR SOURCE
}

\author{
W. E. Olmstead and Catherine A. Roberts \\ Dedicated to the memory of Charles G. Lange. \\ He will be remembered as a good friend and a fine applied mathematician.
}

\begin{abstract}
The possibility of a blow-up solution to the heat equation with a concentrated source in a finite strip is examined. It is found that blow-up always occurs for the Neumann problem, whereas for the Dirichlet problem it depends upon the length of the strip and proximity of the source site to the boundary. For those situations in which blow-up does occur, the growth rate is determined for a certain class of nonlinearities.
\end{abstract}

\section{Introduction}

We examine the explosive behavior of the solution to the heat equation in a onedimensional strip of finite width that contains a concentrated nonlinear source of heat. The problem will be treated for both the Neumann and Dirichlet type boundary conditions. Our investigation is carried out by converting the parabolic initial-boundaryvalue problem to a nonlinear integral equation, which determines the temperature at the site of the concentrated source. The resulting nonlinear Volterra equation of the second kind lends itself to analytical techniques developed in [6], [7].

Our analysis will show that the Neumann problem always leads to a blow-up solution. For the Dirichlet problem, whether or not a blow-up occurs depends upon the parameters of the problem. In particular, it is found that blow-up can always be prevented by locating the concentrated source sufficiently close to an edge of the domain. In those situations where blow-up does occur, for either the Neumann or Dirichlet problem, we will use asymptotic methods to determine the blow-up growth rate of the solution for a variety of nonlinear source functions.

Consider the nonlinear heat equation

$$
\frac{\partial v}{\partial t}(x, t)=\frac{\partial^{2} v}{\partial x^{2}}(x, t)+F[v(x, t), x], \quad 0<x<\ell, t>0
$$

for the temperature $v(x, t)$ in a finite strip. There has been extensive research on blowup solutions for this type of parabolic problem when the nonlinearly has no explicit spatial dependence, i.e., $F=F(v)$; see $[1,3]$ for references to such work. While some investigations such as [5] have considered $F=F(v, x)$, there was a required spatial

Received April 21, 1994, revised September 21, 1994.

1991 Mathematics Subject Classification: 35K60, 45D05, 45M05.

Key words and phrases: integral equation, blow-up solution.

The research of the first author was supported in part by NSF Grant DMS-9401016. 
smoothness. The emphasis here is on the situation in which the nonlinear source has a strongly concentrated and localized spatial dependence of the form

$$
F[v(x, t), x]=\delta(x-a) g[v(x, t)], \quad 0<a<\ell,
$$

where $\delta(x-a)$ is the Dirac delta distribution. This model is motivated by applications in which the ignition of a combustible medium is accomplished by the use of either a heated wire or a pair of small electrodes to supply a large amount of energy to a very confined area.

For (1)-(2), we impose the initial condition

$$
v(x, 0)=v_{0}(x), \quad 0 \leq x \leq \ell,
$$

and either of the boundary conditions

$$
\begin{aligned}
\frac{\partial v}{\partial x}(0, t) & =\frac{\partial v}{\partial x}(\ell, t)=0, \quad(\text { Neumann problem }) \\
v(0, t) & =v(\ell, t)=0, \quad \text { (Dirichlet problem) }
\end{aligned}
$$

It is typical of explosion models that the nonlinear source function $g(v)$ is smooth and has the properties

$$
g(v)>0, \quad g^{\prime}(v)>0, \quad g^{\prime \prime}(v)>0, \quad v>0
$$

It will be further required that

$$
\kappa \equiv \int_{h_{0}}^{\infty} \frac{d v}{g(v)}<\infty
$$

for some specific $h_{0}>0$.

To investigate a possible blow-up solution of (1)-(6), we will convert this problem to an equivalent integral equation for the temperature at the site of the concentrated source, i.e., $v(a, t)$. As a first step, we introduce an integral representation of (1)-(4) in the form

$$
v(x, t)=\int_{0}^{t} \int_{0}^{\ell} G(x, t \mid \xi, s) F[v(\xi, s), \xi] d \xi d s+\int_{0}^{\ell} G(x, t \mid \xi, 0) v_{0}(\xi) d \xi .
$$

Here $G(x, t \mid \xi, s)$ is the Green's function for the linear heat equation with either Neumann or Dirichlet type boundary conditions. In particular, for the Neumann problem, $G=G_{N}$, where

$$
\begin{aligned}
& G_{N}(x, t \mid \xi, s) \\
& \quad=H(t-s)\left\{\frac{1}{\ell}+\frac{2}{\ell} \sum_{n=1}^{\infty} \cos \left(\frac{n \pi \xi}{\ell}\right) \cos \left(\frac{n \pi x}{\ell}\right) \exp \left[-\frac{n^{2} \pi^{2}}{\ell^{2}}(t-s)\right]\right\} \\
& \quad=\frac{H(t-s)}{2[\pi(t-s)]^{\frac{1}{2}}} \sum_{n=-\infty}^{\infty}\left\{\exp \left[-\frac{(x-\xi-2 n \ell)^{2}}{4(t-s)}\right]+\exp \left[-\frac{(x+\xi-2 n \ell)^{2}}{4(t-s)}\right]\right\}
\end{aligned}
$$


while for the Dirichlet problem, $G=G_{D}$, where

$$
\begin{aligned}
& G_{D}(x, t \mid \xi, s) \\
& \quad=H(t-s)\left\{\frac{2}{\ell} \sum_{n=1}^{\infty} \sin \left(\frac{n \pi \xi}{\ell}\right) \sin \left(\frac{n \pi x}{\ell}\right) \exp \left[-\frac{n^{2} \pi^{2}}{\ell^{2}}(t-s)\right]\right\} \\
& \quad=\frac{H(t-s)}{2[\pi(t-s)]^{\frac{1}{2}}} \sum_{n=-\infty}^{\infty}\left\{\exp \left[\frac{(x-\xi-2 n \ell)^{2}}{4(t-s)}\right]-\exp \left[\frac{(x+\xi-2 n \ell)^{2}}{4(t-s)}\right]\right\} .
\end{aligned}
$$

Each Green's function has been expressed in spectral as well as image representation (see [8]), since both forms will be used in the analysis to follow.

Substitution of the expression (2) for the source function in terms of the delta distribution into (7), and evaluation of (7) at $x=a$ yields

$$
v(a, t)=\int_{0}^{t} G(a, t \mid a, s) g[v(a, s)] d s+h(t), \quad t \geq 0 .
$$

Here $h(t)$ is defined in terms of the initial data by

$$
h(t)=\int_{0}^{\ell} G(a, t \mid \xi, 0) v_{0}(\xi) d \xi
$$

with requirements that it is sufficiently smooth and

$$
h^{\prime}(t) \geq 0, \quad 0<h_{0} \leq h(t) \leq h_{\infty}<\infty .
$$

Thus, the initial-boundary-value problem (1)-(4) has been reduced to the nonlinear Volterra equation (10) for $v(a, t)$. The kernel $G(a, t \mid a, s)$ follows from (8) for the Neumann problem and from (9) for the Dirichlet problem. In the analysis to follow, we will investigate (10) for blow-up solutions and the growth rate of such blow-up.

\section{Criteria for blow-up}

To analyze (10) for possible solutions, it is convenient to express that integral equation in the form

$$
u(t)=T u(t) \equiv \int_{0}^{t} k(t-s) g[u(s)+h(s)] d s, \quad t \geq 0,
$$

where we have defined

$$
u(t) \equiv v(a, t)-h(t) .
$$

The form of the kernel $k(t-s)$ depends upon the choice of boundary conditions (4). For the Neumann problem, it follows from (8) that

$$
\begin{aligned}
k(t-s) & =k_{N}(t-s) \\
& =\frac{1}{\ell}+\frac{2}{\ell} \sum_{n=1}^{\infty} \cos ^{2}\left(\frac{n \pi a}{\ell}\right) \exp \left[-\frac{n^{2} \pi^{2}}{\ell^{2}}(t-s)\right] \\
& =\frac{1}{2[\pi(t-s)]^{\frac{1}{2}}} \sum_{n=-\infty}^{\infty}\left\{\exp \left[-\frac{n^{2} \ell^{2}}{(t-s)}\right]+\exp \left[-\frac{(a-n \ell)^{2}}{(t-s)}\right]\right\},
\end{aligned}
$$


while for the Dirichlet problem, it follows from (9) that

$$
\begin{aligned}
k(t-s) & =k_{D}(t-s) \\
& =\frac{2}{\ell} \sum_{n=1}^{\infty} \sin ^{2}\left(\frac{n \pi a}{\ell}\right) \exp \left[-\frac{n^{2} \pi^{2}}{\ell^{2}}(t-s)\right] \\
& =\frac{1}{2[\pi(t-s)]^{\frac{1}{2}}} \sum_{n=-\infty}^{\infty}\left\{\exp \left[-\frac{n^{2} \ell^{2}}{(t-s)}\right]-\exp \left[-\frac{(a-n \ell)^{2}}{(t-s)}\right]\right\}
\end{aligned}
$$

Our analysis of (13) will utilize the techniques of [6]. First, contraction mapping arguments will be employed to establish the existence of a continuous, non-negative, increasing solution for $0 \leq t<t^{*}$. If $t^{*}<\infty$, then certain inequality arguments can be applied to (13) to establish the non-existence of a continuous, non-negative solution for $t \geq t^{* *}>t^{*}$. Thus, there is implied a blow-up as $t \rightarrow \hat{t}, t^{*} \leq \hat{t} \leq t^{* *}<\infty$.

The positivity of any solution to (13) is clear in view of the positivity of $k(t-s)$, $h(t)$, and $g(v)$. To see that any solution also must be increasing, differentiate (13) to obtain

$$
u^{\prime}(t)=k(t) g\left(h_{0}\right)+\int_{0}^{t} k(t-s) g^{\prime}[u(s)+h(s)]\left[u^{\prime}(s)+h^{\prime}(s)\right] d s .
$$

Since $u(t)>0$ for $t>0$, then $u^{\prime}(t)>0$ at least on some small interval, $0<t<\bar{t}$. Furthermore, it is impossible for $u^{\prime}(\bar{t})=0$, because an evaluation of (17) at $t=\bar{t}$ would yield that the right-side is positive. Thus $u^{\prime}(t)>0$ whenever the solution exists.

The existence and uniqueness of a continuous solution of (13) that satisfies

$$
0 \leq u(t) \leq M<\infty, \quad 0 \leq t<t^{*},
$$

follows from the application of contraction mapping arguments. Those arguments require that the integral operator $T$ maps the space of continuous functions satisfying (18) into itself, so that

$$
T u(t) \leq g\left(M+h_{\infty}\right) I(t) \leq M, \quad 0 \leq t<t^{*} .
$$

This inequality depends upon

$$
I(t) \equiv \int_{0}^{t} k(t-s) d s
$$

which is an increasing function in either case (15) or (16). For arbitrary $u_{1}(t)$ and $u_{2}(t)$ which are continuous and satisfy (18), the contraction property of $T$ follows from

$$
\sup _{0 \leq t<t^{*}}\left|T u_{1}(t)-T u_{2}(t)\right| \leq g^{\prime}\left(M+h_{\infty}\right) I(t) \sup _{0 \leq t<t^{*}}\left|u_{1}(t)-u_{2}(t)\right|,
$$

provided that

$$
g^{\prime}\left(M+h_{\infty}\right) I(t)<1, \quad 0 \leq t<t^{*} .
$$

The appropriate choice of $M$ to satisfy both (19) and (22) is the smallest solution of

$$
M=g\left(M+h_{\infty}\right) / g^{\prime}\left(M+h_{\infty}\right)
$$


It then follows that the limiting value of $t^{*}$ is determined by considering

$$
I\left(t^{*}\right)=\left[M / g\left(M+h_{\infty}\right)\right] .
$$

This yields a lower bound on the extent of the interval $\left[0, t^{*}\right)$ over which there is existence and uniqueness of a solution to (13).

Whenever the satisfaction of (24) is limited by some $t^{*}<\infty$, then there is the possibility of a blow-up in the solution of (13). We examine this issue by considering the possible non-existence of a continuous solution to (13) for all $t \geq t^{* *}$. Under the assumption that (13) has a continuous solution for $0 \leq t \leq t_{1}$, it follows from (13) that

$$
u(t)=T u(t) \geq J(t) \equiv \int_{0}^{t} k\left(t_{1}-s\right) g[u(s)+h(s)] d s, \quad 0 \leq t \leq t_{1},
$$

since $k\left(t_{1}-s\right) \leq k(t-s)$ for either (15) or (16). It then follows that

$$
J^{\prime}(t)=k\left(t_{1}-t\right) g[u(t)+h(t)] \geq k\left(t_{1}-t\right) g\left[J(t)+h_{0}\right] .
$$

Integration of the differential inequality (26) yields

$$
\int_{h_{0}}^{J\left(t_{1}\right)+h_{0}} \frac{d v}{g(v)} \geq \int_{0}^{t_{1}} k\left(t_{1}-s\right) d s=I\left(t_{1}\right) .
$$

The assumption that $u(t)$ is continuous for $0 \leq t \leq t_{1}$, insures that $J\left(t_{1}\right)<\infty$, and hence (27) implies

$$
\kappa \equiv \int_{h_{0}}^{\infty} \frac{d v}{g(v)}>I\left(t_{1}\right)
$$

Thus, a contradiction of the existence of a continuous solution occurs if $t_{1}=t^{* *}<\infty$ is such that

$$
I\left(t^{* *}\right)=\kappa<\infty
$$

where the boundedness of $\kappa$ has been required in (6).

The existence of a $t^{* *}<\infty$ which satisfies (29) is the essential criterion for blow-up. This relationship represents a balance between the diffusive nature of the kernel, as reflected by $I\left(t^{* *}\right)$, and the constant $\kappa$, which depends upon both the growth of the nonlinearity $g(v)$ and the value of $h_{0}$ as determined from the initial data.

The implication of our analysis is that if there is blow-up, i.e., $u(t) \rightarrow \infty$ as $t \rightarrow \hat{t}$, then

$$
0<t^{*}<\hat{t}<t^{* *}<\infty
$$

where $t^{*}$ and $t^{* *}$ are determined by (24) and (29), respectively. We will examine this possibility for both the Neumann and Dirichlet problems.

For the Neumann problem, we use the spectral representation of the kernel in (15) to obtain

$$
I_{N}(t)=\int_{0}^{t} k_{N}(t-s) d s=\frac{t}{\ell}+\frac{2 \ell}{\pi^{2}} \sum_{n=1}^{\infty} \frac{1}{n^{2}} \cos ^{2}\left(\frac{n \pi a}{\ell}\right)\left[1-\exp \left(-\frac{n^{2} \pi^{2} t}{\ell^{2}}\right)\right] .
$$


It is clear that $I_{N}(t) \rightarrow \infty$ as $t \rightarrow \infty$, and hence (29) will eventually be satisfied. That is, these always exists some $t^{* *}<\infty$ such that

$$
I_{N}\left(t^{* *}\right)=\kappa
$$

Thus, for the Neumann problem, we always have blow-up.

For the Dirichlet problem, we use the spectral representation of the kernel (16) to obtain

$$
\begin{aligned}
I_{D}(t) & =\int_{0}^{t} k_{D}(t-s) d s=\frac{2 \ell}{\pi^{2}} \sum_{n=1}^{\infty} \frac{1}{n^{2}} \sin ^{2}\left(\frac{n \pi a}{\ell}\right)\left[1-\exp \left(-\frac{n^{2} \pi^{2} t}{\ell^{2}}\right)\right] \\
& =\frac{a(\ell-a)}{\ell}-\frac{2 \ell}{\pi^{2}} \sum_{n=1}^{\infty} \frac{1}{n^{2}} \sin ^{2}\left(\frac{n \pi a}{\ell}\right) \exp \left(-\frac{n^{2} \pi^{2} t}{\ell^{2}}\right) .
\end{aligned}
$$

In this case, it is possible to fulfill the contraction mapping requirements of (19) and (22) with $t^{*}=\infty$ by satisfying

$$
\frac{a(\ell-a)}{\ell}<\frac{1}{g^{\prime}\left(M+h_{\infty}\right)}=\frac{M}{g\left(M+h_{\infty}\right)} .
$$

We can always satisfy (34) by placing the concentrated source close enough to either edge of the strip, so that $a(\ell-a)$ is sufficiently small. This guarantees a unique, continuous solution of (13) for all $t>0$, and hence no blow-up.

On the other hand, it is possible to fulfill the blow-up criterion (29) whenever the parameters are such that

$$
\frac{a(\ell-a)}{\ell}>\kappa \text {. }
$$

For example, it is possible to satisfy (35) by placing the concentrated source at the center $(a=\ell / 2)$ of a sufficiently long strip. Then there exists a $t^{* *}<\infty$ such that

$$
I_{D}\left(t^{* *}\right)=\kappa,
$$

and hence blow-up. Thus, for the Dirichlet problem, we find that blow-up either may or may not occur. If (34) is satisfied, there is no blow-up; while if (35) is satisfied, there is blow-up.

\section{Growth rate at blow-up}

It is assumed here that we have the case, for either the Neumann or the Dirichlet problem, in which there is blow-up. That is, (13) has a unique solution and

$$
u(t) \rightarrow \infty, \quad \text { as } t \rightarrow \hat{t}<\infty .
$$

Based upon this assumption, we will develop a self-consistent asymptotic analysis of (13) that determines the leading-order behavior of $u(t)$ as $t \rightarrow \hat{t}$ for a class of nonlinear source functions $g(v)$ which arise in various applications. In particular, we consider $g(v)$ which satisfy (5) and are certain special cases of those nonlinearities which have the asymptotic growth property

$$
g(v) \sim v^{m}(\log v)^{-n} \exp \left(v^{p}\right) \quad \text { as } v \rightarrow \infty
$$

with $m, n, p \geq 0$. 
It should be mentioned that our analysis does not determine the explicit value of the blow-up point $\hat{t}$. The analysis of Section 2 provides criteria for its existence, as well as the bounds $t^{*} \leq \hat{t} \leq t^{* *}$. In general, numerical methods are required to determine $\hat{t}$.

Under the assumption that (37) holds, we introduce the transformation,

$$
\eta=(\hat{t}-t)^{-1}-\eta_{0}, \quad \eta_{0}=(\hat{t})^{-1}, \quad w(\eta)=u(t) .
$$

Thus, the blow-up behavior (37) becomes

$$
w(\eta) \rightarrow \infty, \quad \text { as } \quad \eta \rightarrow \infty .
$$

The transformation (39) converts (13) to the form

$$
w(\eta)=\int_{0}^{\eta} k\left\{\left(\eta-\eta^{\prime}\right)\left[\left(\eta^{\prime}+\eta_{0}\right)\left(\eta+\eta_{0}\right)\right]^{-1}\right\} \varphi\left(\eta^{\prime}\right) d \eta^{\prime}, \quad \eta \geq 0
$$

where

$$
\varphi(\eta)=\left(\eta+\eta_{0}\right)^{-2} g\left\{w(\eta)+h\left[\hat{t}-\left(\eta+\eta_{0}\right)^{-1}\right]\right\} .
$$

The advantage of this formulation is that certain techniques developed in [2] can be utilized for the asymptotic evaluation, as $\eta \rightarrow \infty$, of integrals like that found in (41). Those techniques have been employed effectively on related problems in $[4,7]$.

Following the methods of [2], let $\eta^{\prime}=\eta \tau$, so that (41) becomes

$$
w(\eta)=\eta I(\eta)
$$

where

$$
I(\eta)=\int_{0}^{1} k\left\{\eta(1-\tau)\left[\left(\eta \tau+\eta_{0}\right)\left(\eta+\eta_{0}\right)\right]^{-1}\right\} \varphi(\eta \tau) d \tau .
$$

Since our interest is in an asymptotic solution of (43) as $\eta \rightarrow \infty$, we will utilize the specific forms of the kernel to simplify (44). It follows from the image representation of the kernels in (15) and (16) that

$$
\begin{aligned}
I(\eta)= & \left(\frac{\eta+\eta_{0}}{\pi \eta}\right)^{\frac{1}{2}}\left\{\frac{1}{2} \int_{0}^{1}(1-\tau)^{-\frac{1}{2}} \Phi(\eta \tau) d \tau\right. \\
& +\sum_{n=1}^{\infty} \int_{0}^{1}(1-\tau)^{-\frac{1}{2}} \exp \left[-\frac{n^{2} \ell^{2}\left(\eta+\eta_{0}\right)\left(\eta \tau+\eta_{0}\right)}{\eta(1-\tau)}\right] \Phi(\eta \tau) d \tau \\
& \left. \pm \frac{1}{2} \sum_{n=-\infty}^{\infty} \int_{0}^{1}(1-\tau)^{-\frac{1}{2}} \exp \left[-\frac{(a-n \ell)^{2}\left(\eta+\eta_{0}\right)\left(\eta \tau+\eta_{0}\right)}{\eta(1-\tau)}\right] \Phi(\eta \tau) d \tau\right\}
\end{aligned}
$$

where

$$
\begin{aligned}
\Phi(\eta \tau) & =\left(\eta \tau+\eta_{0}\right)^{\frac{1}{2}} \varphi(\eta \tau) \\
& =\left(\eta \tau+\eta_{0}\right)^{-\frac{3}{2}} g\left\{w(\eta \tau)+h\left[\hat{t}-\left(\eta \tau+\eta_{0}\right)^{-1}\right]\right\} .
\end{aligned}
$$

In (45), the \pm selection corresponds to $k=k_{N}$ and $k=k_{D}$, respectively. 
It can be shown that the exponential terms in (45) are small, as $\eta \rightarrow \infty$, compared to the one non-exponential term. This is seen by considering a representative exponential term in the limiting form

$$
R(\eta)=\lim _{\varepsilon \rightarrow 0} \int_{\varepsilon}^{1}(1-\tau)^{-\frac{1}{2}} \exp \left[-\frac{\beta\left(\eta+\eta_{0}\right)\left(\eta \tau+\eta_{0}\right)}{\eta(1-\tau)}\right] \Phi(\eta \tau) d \tau, \quad \beta>0 .
$$

It follows that

$$
R(\eta) \leq \lim _{\varepsilon \rightarrow 0}\left\{\left[\int_{\varepsilon}^{1}(1-\tau)^{-\frac{1}{2}} \Phi(\eta \tau) d \tau\right] e^{-\beta \eta \varepsilon}\right\} .
$$

By selecting $\varepsilon=\eta^{-\frac{1}{2}}$, as $\eta \rightarrow \infty$, it is clear that terms of the form (47) are exponentially small compared to that with $\beta=0$.

Thus, we see that, for the asymptotic analysis of (43), it is appropriate to consider

$$
I(\eta) \sim \frac{1}{2} \int_{0}^{1}[\pi(1-\tau)]^{-\frac{1}{2}} \Phi(\eta \tau) d \tau, \quad \eta \rightarrow \infty .
$$

By using this leading-order behavior of $I(\eta)$ in (43), we will be able to determine the growth of $w(\eta)$ near blow-up for certain nonlinear source functions in the form of (38).

To investigate the asymptotic behavior of $I(\eta)$ as $\eta \rightarrow \infty$, the technique of [2] is to employ the Parseval formula for Mellin transforms to obtain

$$
\begin{aligned}
I(\eta) & \sim \frac{1}{2} \int_{0}^{\infty}\left\{[\pi(1-\tau)]^{-\frac{1}{2}} H(1-\tau)\right\} \Phi(\eta \tau) d \tau \\
& \left.=\frac{1}{4 \pi i} \int_{c-i \infty}^{c+i \infty} M\left[\pi^{-\frac{1}{2}}(1-\tau)^{-\frac{1}{2}} H(1-\tau) ; 1-z\right] M[\Phi(\eta \tau) ; z)\right] d z
\end{aligned}
$$

where the Mellin transform is defined by

$$
M[v(\tau) ; z] \equiv \int_{0}^{\infty} \tau^{z-1} v(\tau) d \tau .
$$

In (50), the vertical path of integration in the complex $z$-plane lies within the common strip of analyticity for the two Mellin transforms.

Further simplification of (50) follows from

$$
M\left[\pi^{-\frac{1}{2}}(1-\tau)^{-\frac{1}{2}} H(1-\tau) ; 1-z\right]=\frac{\Gamma(1-z)}{\Gamma\left(\frac{3}{2}-z\right)}
$$

and

$$
M[\Phi(\eta \tau) ; z]=\eta^{-z} M[\Phi(\tau) ; z] .
$$

Thus, it follows that, in place of (43), we can consider the asymptotic equation

$$
w(\eta) \sim \frac{\eta}{4 \pi i} \int_{c-i \infty}^{c+i \infty} \eta^{-z} \frac{\Gamma(1-z)}{\Gamma\left(\frac{3}{2}-z\right)} M[\Phi(\tau) ; z] d z, \quad \eta \rightarrow \infty .
$$

Our analysis of (54) will seek to determine a self-consistent match of the leading-order behavior of each side of this asymptotic equality. 
In order to proceed with the asymptotic analysis of (54), we need more specific information about $M[\Phi ; z]$. That desired information is determined by the asymptotic behavior of $\Phi(\eta)$ as $\eta \rightarrow \infty$. From (46),

$$
\begin{aligned}
\Phi(\eta) & =\left(\eta+\eta_{0}\right)^{-\frac{3}{2}} g\left\{w(\eta)+h\left[\hat{t}-\left(\eta+\eta_{0}\right)^{-1}\right]\right\} \\
& \sim \eta^{-\frac{3}{2}} g[w(\eta)], \quad \text { as } \eta \rightarrow \infty .
\end{aligned}
$$

We will examine (55) for certain special cases of the nonlinear dependence in the form of $(38)$.

We first consider the case of a combined algebraic and logarithmic nonlinearity of the form

$$
g(w) \sim w^{m}(\log w)^{-n}, \quad m>1, \quad n \geq 0, \quad \text { as } w \rightarrow \infty .
$$

For this case, we assume that

$$
w(\eta) \sim A \eta^{r}(\log \eta)^{k}, \quad r>0, \quad \text { as } \eta \rightarrow \infty,
$$

where $A, r$, and $k$ are to be determined. It follows that

$$
\Phi(\eta) \sim A^{m} r^{-n} \eta^{m r-\frac{3}{2}}(\log \eta)^{k m-n}, \quad \text { as } \eta \rightarrow \infty .
$$

For $2 m r>1$ and $k m-n=N=0,1,2, \ldots$, it follows from [2] that $M[\Phi ; z]$ has a pole of order $N+1$ at $z=\frac{3}{2}-m r$, so that

$$
M[\Phi ; z] \sim \frac{(-1)^{N+1} A^{m} r^{-n} N !}{\left[z-\left(\frac{3}{2}-m r\right)\right]^{N+1}}, \quad \text { as } z \rightarrow \frac{3}{2}-m r<1 .
$$

To compute the leading asymptotic contribution from the integral in (54), the vertical path is displaced to the right. In doing so, the pole implied by (59) is encountered before the simple pole of $\Gamma(1-z)$ at $z=1$. Thus, (54) takes the form

$$
A \eta^{r}(\log \eta)^{k} \sim \frac{A^{m} \Gamma\left(m r-\frac{1}{2}\right)}{2 r^{n} \Gamma(m r)} \eta^{m r-\frac{1}{2}}(\log \eta)^{k m-n}, \quad \text { as } \eta \rightarrow \infty .
$$

Matching the two sides of (60) yields

$$
\begin{gathered}
r=\frac{1}{2(m-1)}, \quad k=\frac{n}{m-1}, \\
A=\left\{\frac{2 \Gamma\left[\frac{m}{2(m-1)}\right]}{[2(m-1)]^{n} \Gamma\left[\frac{1}{2(m-1)}\right]}\right\}^{\frac{1}{m-1}},
\end{gathered}
$$

with the restriction that

$$
\frac{n}{m-1}=N=0,1,2, \ldots
$$

Thus, we have found that when the nonlinear source function behaves like (56) with the restriction (62), then

$$
w(\eta) \sim A \eta^{1 / 2(m-1)}(\log \eta)^{n /(m-1)}, \quad \text { as } \eta \rightarrow \infty .
$$


We next consider the case of a combined algebraic and exponential nonlinearity of the form

$$
g(w) \sim w^{m} \exp \left(w^{p}\right), \quad m \geq 0, \quad p>0, \quad \text { as } w \rightarrow \infty .
$$

For this case, we assume

$$
w(\eta) \sim\left[\log \left(A \eta^{\frac{1}{2}}\right)\right]^{\frac{1}{p}} \sim\left[\frac{1}{2} \log \eta+\log A\right]^{\frac{1}{p}}, \quad \text { as } \eta \rightarrow \infty .
$$

Here $A$ is to be determined as well as a restriction on $p$ and $m$ that must hold. It follows that

$$
\Phi(\eta) \sim A\left(\frac{1}{2}\right)^{\frac{m}{p}} \eta^{-1}(\log \eta)^{\frac{m}{p}}, \quad \text { as } \eta \rightarrow \infty .
$$

In order to obtain an asymptotic balance in (54), it is found necessary to take $m / p=$ $N=0,1,2, \ldots$ It then follows that $M[\Phi ; z]$ has a pole of order $N+1$ at $z=1$, so that

$$
M[\Phi ; z] \sim \frac{(-1)^{N+1} A\left(\frac{1}{2}\right)^{\frac{m}{p}} N !}{(z-1)^{N+1}}, \quad \text { as } z \rightarrow 1 .
$$

To compute the leading asymptotic contribution from the integral in (54), the vertical path is displaced to the right. In this case, the pole at $z=1$ implied by (67) coalesces with that from

$$
\Gamma(1-z) \sim-\frac{1}{z-1}, \quad \text { as } z \rightarrow 1,
$$

to give rise to a pole of order $N+2$ at $z=1$. Thus, (54) takes the form

$$
\left(\frac{1}{2} \log \eta\right)^{\frac{1}{p}} \sim \frac{A}{2(N+1) \Gamma\left(\frac{1}{2}\right)}(\log \eta)^{\frac{m}{p}+1}, \quad \text { as } \eta \rightarrow \infty .
$$

Matching the two sides of (69) yields

$$
m+p=1, \quad A=\left(\frac{1}{2}\right)^{\frac{1}{p}-1}(N+1) \Gamma\left(\frac{1}{2}\right)=\left(\frac{1}{2}\right)^{\frac{1}{p}-1} \frac{\pi^{\frac{1}{2}}}{p}
$$

with the restriction that

$$
m=\frac{N}{N+1}, \quad p=\frac{1}{N+1}, \quad N=0,1,2, \ldots .
$$

Thus we have found that when the nonlinear source function behaves like (64) with the restriction (71), then

$$
w(\eta) \sim\left(\frac{1}{2} \log \eta\right)^{\frac{1}{p}}, \quad \text { as } \eta \rightarrow \infty .
$$

Here we have dropped the contribution from the constant $A$ which is implied by (65), because a more complete asymptotic analysis beyond the leading order might well yield additional constant terms. 


\section{Summary and conclusions}

Our analysis of the nonlinear parabolic problem described by (1)-(6) reveals that boundary conditions play a crucial role in the occurrence of blow-up. It was found that blow-up always occurs in the Neumann problem; whereas, it can always be prevented in the Dirichlet problem by locating the concentrated source sufficiently close to an edge. It was further determined that when blow-up does occur, the asymptotic growth is the same for both the Neumann and Dirichlet problems.

For the Neumann problem, there is always blow-up and an upper bound $t^{* *}$ on its occurrence follows from (31) as

$$
\frac{t^{* *}}{\ell}+\frac{2 \ell}{\pi^{2}} \sum_{n=1}^{\infty} \frac{1}{n^{2}} \cos ^{2}\left(\frac{n \pi a}{\ell}\right)\left[1-\exp \left(-\frac{n^{2} \pi^{2} t^{* *}}{\ell^{2}}\right)\right]=\int_{h_{0}}^{\infty} \frac{d v}{g(v)}<\infty
$$

For the Dirichlet problem, it follows from (33) that no blow-up will occur if

$$
\frac{a(\ell-a)}{\ell}<\frac{1}{g^{\prime}\left(M+h_{\infty}\right)}=\frac{M}{g\left(M+h_{\infty}\right)} .
$$

This inequality can always be achieved by placing the concentrated source sufficiently close to one of the edges of the strip. On the other hand, it follows from (35)-(36) there will be a blow-up, with an upper bound $t^{* *}$ on its occurrence, whenever the quantity $a(\ell-a) / \ell$ is such that

$$
\frac{2 \ell}{\pi^{2}} \sum_{n=1}^{\infty} \frac{1}{n^{2}} \sin ^{2}\left(\frac{n \pi a}{\ell}\right) \exp \left(-\frac{n^{2} \pi^{2} t^{* *}}{\ell^{2}}\right)=\frac{a(\ell-a)}{\ell}-\int_{h_{0}}^{\infty} \frac{d v}{g(v)}>0 .
$$

A lower bound $t^{*}$ on the occurrence of blow-up follows from (23) for the Neumann problem as

$$
\frac{t^{*}}{\ell}+\frac{2 \ell}{\pi^{2}} \sum_{n=1}^{\infty} \frac{1}{h^{2}} \cos ^{2}\left(\frac{n \pi a}{\ell}\right)\left[1-\exp \left(-\frac{n^{2} \pi^{2} t^{*}}{\ell^{2}}\right)\right]=\frac{M}{g\left(M+h_{\infty}\right)},
$$

and for the Dirichlet problem as

$$
\frac{a(\ell-a)}{\ell}-\frac{2 \ell}{\pi^{2}} \sum_{n=1}^{\infty} \frac{1}{n^{2}}\left(\frac{n \pi a}{\ell}\right) \exp \left(-\frac{n^{2} \pi^{2} t^{*}}{\ell^{2}}\right)=\frac{M}{g\left(M+h_{\infty}\right)} .
$$

If blow-up does occur as $t \rightarrow \hat{t}, t^{*} \leq \hat{t} \leq t^{* *}$, the leading-order asymptotic growth is the same for both the Neumann and Dirichlet problems. When the nonlinearity is of the form

$$
g(u) \sim u^{m}(\log u)^{-n}, \quad m>1, \quad n \geq 0, \quad \text { as } u \rightarrow \infty
$$

then

$$
u(t) \sim\left\{\frac{2 \Gamma\left[\frac{m}{2(m-1)}\right]}{[2(m-1)]^{n} \Gamma\left[\frac{1}{2(m-1)}\right](\hat{t}-t)^{\frac{1}{2}}}\right\}^{\frac{1}{m-1}}\left[\log \frac{1}{\hat{t}-t}\right]^{\frac{n}{m-1}}, \quad \text { as } t \rightarrow \hat{t}
$$

with the restriction that

$$
\frac{n}{m-1}=N=0,1,2, \ldots
$$


When the nonlinearity is of the form

$$
g(u) \sim u^{m} \exp \left(u^{p}\right), \quad m \geq 0, \quad p>0, \quad \text { as } u \rightarrow \infty,
$$

then

$$
u(t) \sim\left(\frac{1}{2} \log \frac{1}{\hat{t}-t}\right)^{\frac{1}{p}}, \quad \text { as } t \rightarrow \hat{t}
$$

with the restriction that

$$
m=1-p, \quad p=\frac{1}{N+1}, \quad N=0,1,2, \ldots .
$$

\section{References}

1. J. Bebernes and D. Eberly, Mathematical Problems from Combustion Theory, Springer-Verlag, New York, 1989.

2. N. Bleistein and R. A. Handelsman, Asymptotic Expansion of Integrals, Holt, Rinehardt and Winston, New York, 1975.

3. H. A. Levine, The role of critical exponents in blow-up theorems, SIAM Rev. 32 (1990), 262-288.

4. W. E. Olmstead and R. A. Handelsman, Asymptotic solution to a class of nonlinear Volterra integral equations II, SIAM J. Appl. Math. 30 (1976), 180-189.

5. C. V. Pao, Asymptotic behavior and nonexistence of global solutions for a class of nonlinear boundary value problems of parabolic type, J. Math. Anal. Appl. 65 (1978), 616-637.

6. C. A. Roberts, D. G. Lasseigne, and W. E. Olmstead, Volterra equations which model explosion in a diffusive medium, J. Integral Eqns. and Appls. 5 (1993), 531-546.

7. C. A. Roberts and W. E. Olmstead, Growth rates for blow-up solutions of nonlinear Volterra equations, Q. of Appl. Math. (to appear).

8. I. Stakgold, Boundary Value Problems of Mathematical Physics, Macmillan, New York, 1968.

Department of Engineering Sciences and Applied Mathematics, Northwestern University, EVANSTON, IL 60208, U.S.A.

Department of Mathematics, University of Rhode Island, Kingston, Ri 02881, U.S.A. 